\title{
Stability and absorbing set of parabolic chemotaxis model of Escherichia coli*
}

\author{
Salvatore Rionero ${ }^{\mathrm{a}}$, Maria Vitiello ${ }^{\mathrm{b}, 1}$ \\ ${ }^{a}$ Department of Mathematics and Applications R. Caccioppoli \\ University of Naples Federico II \\ Via Cintia, Monte S. Angelo, 80126, Naples, Italy \\ rionero@unina.it \\ ${ }^{\mathrm{b}}$ Department of Mechanics, Mathematics and Management \\ Politecnico di Bari, \\ Via Orabona, 70125, Bari, Italy \\ vitiello@poliba.it
}

Received: 2 May 2012 / Revised: 26 February 2013 / Published online: 18 April 2013

Abstract. This paper is devoted to model (1) for escherichia coli, introduced in [1]. Based on the experimental observations of Budrene and Berg [2,3], Tyson and coworkers derived (1) with $n$ cell density, $c$ chemotrattactant concentration and $s$ stimulant concentration. Our aim is to study the stability of constant meaning full solution and ultimately boundedness of the solutions. Precisely:

(i) linear and nonlinear stability is proved by using a peculiar Lyapunov function,

(ii) the ultimately boundedness of the solutions in the $L^{2}$-norm is obtained,

(iii) conditions guaranteeing the global stability are also obtained.

Keywords: chemotaxis model, linear and nonlinear stability, absorbing set.

\section{Introduction}

Mathematical models of the biological systems are an important tool. Much attention has been paid to pattern formation in the nature world. Especially, patterns of bacteria colonies have long been investigated, and mathematical models of pattern formation have been developed extensively. For an account of the state of art of chemotaxis and chemotaxis models we refer to [4-7] and references therein.

The collection of diverse patterns observed by Budrene and Berg [2,3] is an interesting and well-documented example of complex pattern formation by bacteria. The most complex patterns are formed by Escherichia coli in semi-solid medium. These models are

\footnotetext{
* This work has been performed under the auspices of the GNFM of INDAM and was supported in part from the Leverhulme Trust, "Tipping points: mathematics, metaphors and meanings".

${ }^{1}$ Corresponding author.
} 
time-dependent systems of partial differential equations, typically in two or three space dimensions, which contain three distinct sets of terms modelling three distinct processes: reaction term, diffusion term and chemotaxis term. Reaction diffusion equations including only the first two processes above have been widely studied, both theoretically and numerically, as models of many biological systems $[6,8,9]$ (cfr. also [10,11]).

Here, we describe dynamical behaviour of E. coli bacterial chemotaxis, the best understood phenomena among pattern process in bioscience. A small initial inoculum of bacteria forms a swarm ring of high cell density which expands outward from the inoculum. The patterns are formed by fully-motile cells, but a large portion of the cells becomes non-motile for some unknown reason, and these maintain the pattern. The E. coli patterns are formed when the bacteria are exposed to intermediates of the tricarboxylic acid (TCA) cycle, principally succinate. In response the cell secrete aspartate, which is a potent chemoattractant. A chemoattractant for E. coli is a chemical which the bacteria like, in the sense that the bacterial cells tend to move up concentration gradients of the chemical, a process known as chemotaxis.

We choose a parabolic PDE model of reaction diffusion type which is modified from $[1,12]$. A model proposed by Tyson et al. [1] contains most of the relevant features observed in E. coli chemotaxis. Tyson and coworkers based their model on the experimental observations of Budrene and Berg [2,3] and they derived the following mathematical representation with three variables the cell density $n$, the chemoattractant concentration $c$ and the stimulant concentration $s$

$$
\left\{\begin{array}{l}
n_{t}=d_{n} \Delta n-\nabla\left[\frac{k_{1} n}{\left(k_{2}+c\right)^{2}} \nabla c\right]+k_{3} n\left(\frac{k_{4} s^{2}}{k_{9}+s^{2}}-n\right) \\
c_{t}=d_{c} \Delta c+k_{5} s \frac{n^{2}}{k_{6}+n^{2}}-k_{7} n c \\
s_{t}=d_{s} \Delta s-k_{8} n \frac{s^{2}}{k_{9}+s^{2}} .
\end{array}\right.
$$

The first term in (1) describes Fickian diffusion. In $(1)_{1}$, the second term denotes the chemotactic response and the third is for the growth of cells. Similarly, the production of chemoattractant (second term in $(1)_{2}$ adds to its diffusion transport, while the uptake of chemoattractant by the cells (third term) reduces its extra cellular concentration. The rate of change of nutrient concentration $(1)_{3}$ is difference between the rates of diffusion and consumption.

Tyson applied two simplifications: the succinate concentration $s$, was assumed to be constant and used as a parameter, and $k_{7}$ and $k_{8}$ were set to zero. This eliminate equation $(1)_{3}$ and reduces equations $(1)_{1}$ and $(1)_{2}$. All these models contain more or less assumption, yet none has been studied with respect to all three pattern-forming processes, when any realistic model of the system must reproduce them all.

In this paper we present the mathematical model that captures all three observed pattern-forming processes and we analyze the linear and nonlinear $L^{2}$-stability of the solution of (1) under Neumann boundary data by following the methodology formulated in [13-17]. 
The plain of the paper is the following. Section 2 is devoted to some preliminaries. In Section 3, we recall a Lyapunov functional introduced previously in [13] and analyze the linear stability of positive steady states. Section 4 is dedicated to the (local) nonlinear stability. In Section 5 the existence of an absorbing set is shown. In Section 6 conditions for the global stability are derived. The paper ends with an appendix in which the proof of Lemma 1 is sketched.

\section{Preliminaries}

By introducing the scalings [6]

$$
\begin{aligned}
& u=\frac{n}{n_{0}}, \quad v=\frac{c}{k_{2}}, \quad w=\frac{s}{\sqrt{k_{9}}}, \quad t^{*}=k_{7} n_{0} t, \quad \Delta^{*}=\frac{d_{c}}{k_{7} n_{0}} \Delta, \\
& d_{1}=\frac{d_{n}}{d_{c}}, \quad d_{3}=\frac{d_{s}}{d_{c}}, \quad \alpha=\frac{k_{1}}{d_{c} k_{2}}, \quad \rho=\frac{k_{3}}{k_{7}}, \\
& \delta=\frac{k_{4}}{n_{0}}, \quad \beta=\frac{k_{5} \sqrt{k_{9}}}{k_{7} k_{2} n_{0}}, \quad k=\frac{k_{8}}{k_{7} \sqrt{k_{9}}}, \quad \mu=\frac{k_{6}}{n_{0}^{2}},
\end{aligned}
$$

the mathematical model, dropping the stars, in dimensionless form is

$$
\left\{\begin{array}{l}
u_{t}=d_{1} \Delta u-\alpha \nabla\left[\frac{u}{(1+v)^{2}} \nabla v\right]+\rho u\left(\frac{\delta w^{2}}{1+w^{2}}-u\right) \\
v_{t}=\Delta v+\beta w \frac{u^{2}}{\mu+u^{2}}-u v \\
w_{t}=d_{3} \Delta w-k u \frac{w^{2}}{1+w^{2}},
\end{array}\right.
$$

where $u$ denotes bacterial cell density, $v$ the aspartate concentration and $w$ the succinate concentration.

We choose $\Omega \subseteq R^{3}$ a bounded smooth domain and we refer here to the positive smooth solutions of (2), under the smooth initial data

$$
u(x, 0)=u_{0}(x), \quad v(x, 0)=v_{0}(x), \quad w(x, 0)=w_{0}(x), \quad x \in \Omega,
$$

and we associate to (2) the Neumann boundary conditions ( $\mathbf{n}$ being the outward normal to $\partial \Omega$ )

$$
\frac{\mathrm{d} u}{\mathrm{~d} \mathbf{n}}=0, \quad \frac{\mathrm{d} v}{\mathrm{~d} \mathbf{n}}=0, \quad \frac{\mathrm{d} w}{\mathrm{~d} \mathbf{n}}=0 \quad \text { on } \partial \Omega \times R^{+}
$$

We denote by

- $\langle\cdot, \cdot\rangle$ the scalar product in $L^{2}(\Omega)$,

- $\|\cdot\|$ the $L^{2}(\Omega)$-norm, 
- $H^{1}(\Omega)$ the Sobolev space such that

$$
\psi \in H^{1}(\Omega) \rightarrow\left\{\psi^{2}+(\nabla \psi)^{2} \in L(\Omega), \frac{\mathrm{d} \psi}{\mathrm{d} \mathbf{n}}=0 \text { on } \partial \Omega\right\}
$$

and recall that in $H^{1}(\Omega)$ holds the inequality [8]

$$
\|\nabla \psi\|^{2} \geqslant \bar{\alpha}\|\psi\|^{2}
$$

with $\bar{\alpha}=\min _{H^{1}(\Omega)}\left(\|\nabla \psi\|^{2} /\|\psi\|^{2}\right)$, i.e. $\bar{\alpha}$ is the lowest nonzero eigenvalue of $\Delta \Psi=-\alpha \Psi$ in $H^{1}(\Omega)$.

Remark 1. In the sequel we will denote by $I_{1}$ the set of solutions of (2) with initial data $\left(0, v_{0}, w_{0}\right)$ and by $I_{2}$ the set of solutions with initial data $\left(u_{0}, v_{0}, w_{0}\right)$ with $u_{0} \neq$ const.

\section{Equilibria and stability}

In this section we analyze the equilibrium points of the system (2) and their stability. We observe that, by inspection of (2), one deduces that there are only two types of relevant equilibria given by

$$
E_{0}=(0,0,0), \quad E^{*}=\left(0, V^{*}, W^{*}\right)
$$

with $V^{*}$ and $W^{*}$ positive constants.

Let us consider the perturbation to the generic equilibrium $E^{*}$

$$
u=U, \quad v=V^{*}+V, \quad w=W^{*}+W,
$$

the equations governing the perturbation $(U, V, W)$ to the basic state $E^{*}$ are

$$
\left\{\begin{array}{l}
U_{t}=a_{11} U+d_{1} \Delta U+F_{1}, \\
V_{t}=a_{21} U+a_{23} W+\Delta V+F_{2}, \\
W_{t}=a_{31} U+d_{3} \Delta W+F_{3},
\end{array}\right.
$$

where

$$
\left\{\begin{array}{l}
a_{11}=\rho \delta, \quad a_{21}=-V^{*}, \quad a_{23}=\beta, \quad a_{31}=-k \\
F_{1}=-\alpha \nabla\left[\frac{U}{\left[1+\left(V+V^{*}\right)\right]^{2}} \nabla V\right]-\rho U\left[\frac{\delta}{1+\left(W+W^{*}\right)^{2}}+U\right] \\
F_{2}=\beta \mu\left[\frac{U^{2} W^{*}}{\left(\mu+U^{2}\right) \mu}-\frac{W}{\mu+U^{2}}\right]-U V, \quad F_{3}=\frac{k U}{1+\left(W+W^{*}\right)^{2}} .
\end{array}\right.
$$

It can be sketched that the stability of the equilibria depends on the fulfillment of the initial conditions. We observe that if $\left(u_{0}, v_{0}, w_{0}\right) \in I_{1}$ the stability analysis can be easily performed and simple stability is easily obtained. Of course, this is not the situation we are interested in and hence our analysis will be focused on the case $\left(u_{0}, v_{0}, w_{0}\right) \in I_{2}$ 
and study the (asymptotic) stability of the equilibrium $E^{*}$ with respect the perturbation $(U, V, W)^{\mathrm{T}}$ with $U_{0} \neq 0$, i.e., in order to make the analysis consistent with the experiments, we consider initially nonzero cell density, nonzero stimulant concentration and zero concentration of chemoattractant. In fact $v(x, 0)$ is set to zero because, initially, bacteria have not secreted any chemoattractant [12].

In vectorial form, system (5) can be expressed as

$$
\mathbf{U}_{t}=L \mathbf{U}+\mathbf{F} \quad \text { in } \Omega \times R^{+},
$$

with

$$
\mathbf{U}=(U, V, W)^{\mathrm{T}}, \quad \mathbf{F}=\left(F_{1}, F_{2}, F_{3}\right)^{\mathrm{T}}, \quad L=\left(\begin{array}{ccc}
a_{11}+d_{1} \Delta & 0 & 0 \\
a_{21} & d_{2} \Delta & a_{23} \\
a_{31} & 0 & d_{3} \Delta
\end{array}\right)
$$

$a_{i j}=$ const $\in R, d_{i}=$ const $>0, i, j=1,2,3$.

Setting

$$
b_{i i}=a_{i i}-d_{i} \bar{\alpha}, \quad i=1,2,3,
$$

let us consider the system

$$
\frac{\mathrm{d} \mathbf{U}}{\mathrm{d} t}=L \mathbf{U}
$$

with

$$
L=\left(\begin{array}{ccc}
b_{11} & 0 & 0 \\
a_{21} & b_{22} & a_{23} \\
a_{31} & 0 & b_{33}
\end{array}\right)
$$

To the matrix $L$ we will apply the following Lemma.

Lemma 1. The Routh-Hurwitz stability conditions of the matrix

$$
\left(\begin{array}{ccc}
\gamma_{11} & 0 & 0 \\
\gamma_{21} & \gamma_{22} & \gamma_{23} \\
\gamma_{31} & \gamma_{32} & \gamma_{33}
\end{array}\right)
$$

with $\gamma_{i j}$ real entries, are

$$
\gamma_{11}<0, \quad I=\gamma_{22}+\gamma_{33}<0, \quad A=\gamma_{22} \gamma_{33}-\gamma_{23} \gamma_{32}>0 .
$$

Proof. The proof is given in [18]. For the sake of completeness a sketch of the proof is given in the Appendix.

Remark 2. Applying Lemma 1, to (9), it follows that the zero solution of (8) is asymptotically stable if and only if

$$
b_{11}<0, \quad I=b_{22}+b_{33}<0, \quad A=b_{22} b_{33}>0,
$$

and hence, in view of $a_{22}=a_{33}=0$, if and only if

$$
b_{11}<0 \text {. }
$$


Denoting now by $\mu_{i}$ ( to be chosen suitably) positive rescaling constants and setting

$$
\begin{aligned}
& U=\mu_{1} U_{1}, \quad V=\mu_{2} U_{2}, \quad W=\mu_{3} U_{3}, \\
& b_{i j}=\frac{\mu_{j}}{\mu_{i}} a_{i j}, \quad i \neq j, \quad i, j=1,2,3,
\end{aligned}
$$

(5), in view of (7), become

$$
\left\{\begin{array}{l}
\frac{\mathrm{d} U_{1}}{\mathrm{~d} t}=b_{11} U_{1}+d_{1}\left(\Delta U_{1}+\bar{\alpha} U_{1}\right)+\frac{1}{\mu_{1}} F_{1}^{*}, \\
\frac{\mathrm{d} U_{2}}{\mathrm{~d} t}=b_{21} U_{1}+b_{22} U_{2}+b_{23} U_{3}+\left(\Delta U_{2}+\bar{\alpha} U_{2}\right)+\frac{1}{\mu_{2}} F_{2}^{*}, \\
\frac{\mathrm{d} U_{3}}{\mathrm{~d} t}=b_{31} U_{1}+b_{33} U_{3}+d_{3}\left(\Delta U_{3}+\bar{\alpha} U_{3}\right)+\frac{1}{\mu_{3}} F_{3}^{*}
\end{array}\right.
$$

with

$$
F_{i}^{*}=F_{i}\left(\mu_{1} U_{1}, \mu_{2} U_{2}, \mu_{3} U_{3}\right), \quad i=1,2,3 .
$$

To (11) we associate the Lyapunov functional introduced by Rionero [13]

$$
W(t)=\frac{1}{2}\left\|U_{1}\right\|^{2}+V
$$

with

$$
V=\frac{1}{2}\left[A\left(\left\|U_{2}\right\|^{2}+\left\|U_{3}\right\|^{2}\right)+\left\|b_{22} U_{3}-b_{32} U_{2}\right\|^{2}+\left\|b_{23} U_{3}-b_{33} U_{2}\right\|^{2}\right] .
$$

The temporal derivative of $W$ along the solutions of (11) is given by

$$
\dot{W}=b_{11}\left\|U_{1}\right\|^{2}+I A\left(\left\|U_{2}\right\|^{2}+\left\|U_{3}\right\|^{2}\right)+\Phi_{1}+\Phi_{2}+\Phi_{3}
$$

with

$$
\begin{aligned}
\Phi_{1}= & \left(A_{1} b_{21}-A_{3} b_{31}\right)\left\langle U_{1}, U_{2}\right\rangle+\left(A_{2} b_{31}-A_{3} b_{21}\right)\left\langle U_{1}, U_{3}\right\rangle, \\
\Phi_{2}= & \left\langle U_{1}, d_{1}\left(\Delta U_{1}+\bar{\alpha} U_{1}\right)\right\rangle+\left\langle A_{1} U_{2}-A_{3} U_{3}, \Delta U_{2}+\bar{\alpha} U_{2}\right\rangle \\
& +\left\langle A_{2} U_{3}-A_{3} U_{2}, d_{3}\left(\Delta U_{3}+\bar{\alpha} U_{3}\right)\right\rangle, \\
\Phi_{3}= & \frac{1}{\mu_{1}}\left\langle U_{1}, F_{1}^{*}\right\rangle+\frac{1}{\mu_{2}}\left\langle A_{1} U_{2}-A_{3} U_{3}, F_{2}^{*}\right\rangle+\frac{1}{\mu_{3}}\left\langle A_{2} U_{3}-A_{3} U_{2}, F_{3}^{*}\right\rangle
\end{aligned}
$$

and

$$
A_{1}=A+\left(b_{33}\right)^{2}, \quad A_{2}=A+\left(b_{22}\right)^{2}+\left(b_{23}\right)^{2}, \quad A_{3}=b_{23} b_{33} .
$$

Lemma 2. Let

$$
\rho \delta<d_{1} \bar{\alpha}
$$

holds. Then

$$
\Phi_{1} \leqslant \frac{1}{2}\left[\left|b_{11}\right|\left\|U_{1}\right\|^{2}+|I A|\left(\left\|U_{2}\right\|^{2}+\left\|U_{3}\right\|^{2}\right)\right]
$$


Proof. Choosing $\mu_{2}=\mu_{3}=1$, we observe that (6) and (13) imply (10) so that Lemma 3.3 in [13] can be applied. ${ }^{2}$

Then, according to the procedure used in [13], Lemma 3.2, one obtains

Lemma 3. Let

$$
\left(1+d_{3}\right)\left|A_{3}\right|=2 \sqrt{A_{1} A_{2} d_{3}}
$$

holds. Then

$$
\Phi_{2} \leqslant 0
$$

Proof. In view of the boundary conditions it turns out that

$$
\begin{aligned}
\Phi_{2}= & d_{1}\left(-\left\|\nabla U_{1}\right\|^{2}+\bar{\alpha}\left\|U_{1}\right\|^{2}\right)+A_{1}\left(-\left\|\nabla U_{2}\right\|^{2}+\bar{\alpha}\left\|U_{2}\right\|^{2}\right) \\
& +A_{2} d_{3}\left(-\left\|\nabla U_{3}\right\|^{2}+\bar{\alpha}\left\|U_{3}\right\|^{2}\right)+\left(1+d_{3}\right) A_{3}\left\langle\nabla U_{2}, \nabla U_{3}\right\rangle \\
& -\bar{\alpha}\left(1+d_{3}\right) A_{3}\left\langle U_{2}, U_{3}\right\rangle .
\end{aligned}
$$

By virtue of (15) it follows that

$$
\begin{aligned}
\Phi_{2}= & d_{1}\left(-\left\|\nabla U_{1}\right\|^{2}+\bar{\alpha}\left\|U_{1}\right\|^{2}\right) \\
& -\left[\left\|\nabla\left(\sqrt{A_{1}} U_{2} \pm \sqrt{d_{3} A_{2}} U_{3}\right)\right\|^{2}-\bar{\alpha}\left\|\sqrt{A_{1}} U_{2} \pm \sqrt{d_{3} A_{2}} U_{3}\right\|^{2}\right]
\end{aligned}
$$

and hence (4) implies (16).

On linearizing (11), (12) reduces to

$$
\dot{W}=b_{11}\left\|U_{1}\right\|^{2}+I A\left(\left\|U_{2}\right\|^{2}+\left\|U_{3}\right\|^{2}\right)+\Phi_{1}+\Phi_{2}
$$

and the following theorem holds true.

Theorem 1. Let (13) and (15) hold. Then $\left(0, V^{*}, W^{*}\right)$ is linearly asymptotically stable with respect to $L^{2}(\Omega)$-norm.

Proof. In view of Lemma 2 and Lemma 3, it follows that

$$
\dot{W} \leqslant-\frac{1}{2}\left[\left|b_{11}\right|\left\|U_{1}\right\|^{2}+|I A|\left(\left\|U_{2}\right\|^{2}+\left\|U_{3}\right\|^{2}\right)\right] .
$$

\footnotetext{
${ }^{2}$ Setting

it follows that

$$
m=\sup \left(\left|A_{1} a_{21}-A_{3} a_{31}\right|,\left|A_{2} a_{31}-A_{3} a_{21}\right|\right),
$$

$$
\begin{aligned}
\Phi_{1} & \leqslant m \mu_{1}\left(\left\langle\left|U_{1}\right|,\left|U_{2}\right|+\left|U_{3}\right|\right\rangle\right) \leqslant m \mu_{1}\left(\left\|U_{1}\right\|\left(\left\|U_{2}\right\|+\left\|U_{3}\right\|\right)\right) \\
& \leqslant \frac{m^{2} \mu_{1}^{2}}{|I A|}\left\|U_{1}\right\|^{2}+\frac{1}{2}|I A|\left(\left\|U_{2}\right\|^{2}+\left\|U_{3}\right\|^{2}\right)
\end{aligned}
$$
}

and hence

$$
\mu_{1}^{2}=\frac{\left|b_{11} I A\right|}{2 m^{2}} \Longrightarrow \quad(14) .
$$


Further $V$ is equivalent to the $L^{2}$-norm, i.e. exist two positive constants $K_{1}, K_{2}$ such that

$$
K_{1}\left(\left\|U_{2}\right\|^{2}+\left\|U_{3}\right\|^{2}\right) \leqslant V \leqslant K_{2}\left(\left\|U_{2}\right\|^{2}+\left\|U_{3}\right\|^{2}\right)
$$

with

$$
K_{1}=\frac{1}{2} A, \quad K_{2}=\frac{1}{2} A+\left(b_{22}\right)^{2}+\left(b_{23}\right)^{2}+\left(b_{33}\right)^{2} .
$$

By virtue of (18), from (17) it turns out that

$$
\dot{W} \leqslant-\frac{1}{2}\left|b_{11}\right||| U_{1} \|^{2}-\frac{|I A|}{K_{2}} V
$$

i.e.

$$
\dot{W} \leqslant-\delta W
$$

with

$$
\delta=\inf \left(\left|b_{11}\right|, \frac{|I A|}{K_{2}}\right) .
$$

Therefore it follows that

$$
W \leqslant W(0) \mathrm{e}^{-\delta t}
$$

and the linear asymptotic stability is proved.

\section{Nonlinear stability}

Setting

$$
\bar{b}_{i i}=b_{i i}+\bar{\alpha} \epsilon, \quad \bar{d}_{i}=d_{i}-\epsilon, \quad \epsilon=\mathrm{const}>0, i=1,2,3,
$$

it follows that (6) imply

$$
\bar{A}=\bar{b}_{22} \bar{b}_{33}-a_{23} a_{32}>0, \quad \bar{I}=\bar{b}_{22}+\bar{b}_{33}<0 .
$$

By virtue of (19), from (11) one obtains

$$
\left\{\begin{array}{l}
\frac{\mathrm{d} U_{1}}{\mathrm{~d} t}=\bar{b}_{11} U_{1}+G_{1}+\frac{1}{\mu_{1}} F_{1}^{*}, \\
\frac{\mathrm{d} U_{2}}{\mathrm{~d} t}=b_{21} U_{1}+\bar{b}_{22} U_{2}+b_{23} U_{3}+G_{2}+\frac{1}{\mu_{2}} F_{2}^{*}, \\
\frac{\mathrm{d} U_{3}}{\mathrm{~d} t}=b_{31} U_{1}+\bar{b}_{33} U_{3}+G_{3}+\frac{1}{\mu_{3}} F_{3}^{*}
\end{array}\right.
$$

with

$$
G_{i}=\bar{d}_{i}\left(\Delta U_{i}+\bar{\alpha} U_{i}\right)+\epsilon \Delta U_{i}, \quad i=1,2,3 .
$$

Let us define

$$
\bar{W}(t)=\frac{1}{2}\left\|U_{1}\right\|^{2}+\bar{V}
$$


with

$$
\bar{V}=\frac{1}{2}\left[\bar{A}\left(\left\|U_{2}\right\|^{2}+\left\|U_{3}\right\|^{2}\right)+\left\|\bar{b}_{22} U_{3}-b_{32} U_{2}\right\|^{2}+\left\|b_{23} U_{3}-\bar{b}_{33} U_{2}\right\|^{2}\right] .
$$

The time derivative of $\bar{W}$ is given by

$$
\frac{\mathrm{d} \bar{W}}{\mathrm{~d} t}=\bar{b}_{11}\left\|U_{1}\right\|^{2}+\bar{I} \bar{A}\left(\left\|U_{2}\right\|^{2}+\left\|U_{3}\right\|^{2}\right)+\bar{\Phi}_{1}+\bar{\Phi}_{2}+\bar{\Phi}_{3}
$$

with

$$
\begin{aligned}
& \bar{\Phi}_{1}=\left(\bar{A}_{1} b_{21}-\bar{A}_{3} b_{31}\right)\left\langle U_{1}, U_{2}\right\rangle+\left(\bar{A}_{2} b_{31}-\bar{A}_{3} b_{21}\right)\left\langle U_{1}, U_{3}\right\rangle \\
& \bar{\Phi}_{2}=\left\langle U_{1}, G_{1}\right\rangle+\left\langle\bar{A}_{1} U_{2}-\bar{A}_{3} U_{3}, G_{2}\right\rangle+\left\langle\bar{A}_{2} U_{3}-\bar{A}_{3} U_{2}, G_{3}\right\rangle, \\
& \bar{\Phi}_{3}=\frac{1}{\mu_{1}}\left\langle U_{1}, F_{1}^{*}\right\rangle+\frac{1}{\mu_{2}}\left\langle\bar{A}_{1} U_{2}-\bar{A}_{3} U_{3}, F_{2}^{*}\right\rangle+\frac{1}{\mu_{3}}\left\langle\bar{A}_{2} U_{3}-\bar{A}_{3} U_{2}, F_{3}^{*}\right\rangle
\end{aligned}
$$

with

$$
\bar{A}_{1}=\bar{A}+\left(\bar{b}_{33}\right)^{2}, \quad \bar{A}_{2}=\bar{A}+\left(\bar{b}_{22}\right)^{2}+\left(b_{23}\right)^{2}, \quad \bar{A}_{3}=b_{23} \bar{b}_{33} .
$$

Let $\bar{b}_{11}<0$, from Lemma $2, \bar{\Phi}_{1}$ is given by

$$
\bar{\Phi}_{1} \leqslant \frac{1}{2}\left[\left|\bar{b}_{11}\right|\left\|U_{1}\right\|^{2}+|\bar{I} \bar{A}|\left(\left\|U_{2}\right\|^{2}+\left\|U_{3}\right\|^{2}\right)\right] .
$$

Moreover

$$
\begin{aligned}
\bar{\Phi}_{2}= & \left\langle U_{1}, \bar{d}_{1}\left(\Delta U_{1}+\bar{\alpha} U_{1}\right)\right\rangle+\left\langle\bar{A}_{1} U_{2}-\bar{A}_{3} U_{3}, \bar{d}_{2}\left(\Delta U_{2}+\bar{\alpha} U_{2}\right)\right\rangle \\
& +\left\langle\bar{A}_{2} U_{3}-\bar{A}_{3} U_{2}, \bar{d}_{3}\left(\Delta U_{3}+\bar{\alpha} U_{3}\right)\right\rangle+\left\langle U_{1}, \epsilon \Delta U_{1}\right\rangle \\
& +\left\langle\bar{A}_{1} U_{2}-\bar{A}_{3} U_{3}, \epsilon \Delta U_{2}\right\rangle+\left\langle\bar{A}_{2} U_{3}-\bar{A}_{3} U_{2}, \epsilon \Delta U_{3}\right\rangle
\end{aligned}
$$

and following the procedure used in Lemma 3 with

$$
\left(\bar{d}_{2}+\bar{d}_{3}\right)\left|\bar{A}_{3}\right|=2 \sqrt{\bar{A}_{1} \bar{A}_{2} \bar{d}_{2} \bar{d}_{3}}
$$

one obtains

$$
\bar{\Phi}_{2} \leqslant-\epsilon\left\|\nabla U_{1}\right\|^{2}-\epsilon \bar{A}_{1}\left\|\nabla U_{2}\right\|^{2}-\epsilon \bar{A}_{2}\left\|\nabla U_{3}\right\|^{2}+2 \epsilon \bar{A}_{3}\left\langle\nabla U_{2}, \nabla U_{3}\right\rangle .
$$

In view of (22) it follows that

$$
\begin{aligned}
\bar{\Phi}_{2} & \leqslant-\epsilon\left(\left\|\nabla U_{1}\right\|^{2}+\frac{\left(\sqrt{\bar{d}_{2}}-\sqrt{d_{3}}\right)^{2}}{\bar{d}_{2}+\bar{d}_{3}} \bar{A}_{1}\left\|\nabla U_{2}\right\|^{2}+\frac{\left(\sqrt{\bar{d}_{2}}-\sqrt{d_{3}}\right)^{2}}{\bar{d}_{2}+\bar{d}_{3}} \bar{A}_{2}\left\|\nabla U_{3}\right\|^{2}\right) \\
& \leqslant-\epsilon \bar{\delta}\left(\left\|\nabla U_{1}\right\|^{2}+\left\|\nabla U_{2}\right\|^{2}+\left\|\nabla U_{3}\right\|^{2}\right)
\end{aligned}
$$

with

$$
\bar{\delta}=\min \left\{1, \frac{\left(\sqrt{\bar{d}_{2}}-\sqrt{d_{3}}\right)^{2}}{\bar{d}_{2}+\bar{d}_{3}} \bar{A}_{1}, \frac{\left(\sqrt{\bar{d}_{2}}-\sqrt{d_{3}}\right)^{2}}{\bar{d}_{2}+\bar{d}_{3}} \bar{A}_{2}\right\}
$$


In view of (21) and (23) one has

$$
\begin{aligned}
\frac{\mathrm{d} \bar{W}}{\mathrm{~d} t} \leqslant & -\frac{1}{2}\left[\left|\bar{b}_{11}\right|\left\|U_{1}\right\|^{2}+|\bar{I} \bar{A}|\left(\left\|U_{2}\right\|^{2}+\left\|U_{3}\right\|^{2}\right)\right] \\
& -\epsilon \bar{\delta}\left(\left\|\nabla U_{1}\right\|^{2}+\left\|\nabla U_{2}\right\|^{2}+\left\|\nabla U_{3}\right\|^{2}\right)+\bar{\Phi}_{3} .
\end{aligned}
$$

It remains to estimate the term $\bar{\Phi}_{3}$ appearing in (24).

Of course, from the biological point of view, we can confine ourselves to the positive solutions of (20) and hence to perturbations such that

$$
\mu_{3} U_{3}>-W^{*}
$$

Since

$$
\left(U_{3}+W^{*}\right)^{2} \geqslant 0 \rightarrow U_{3}^{2} \geqslant-W^{*}-2 U_{3} W^{*}
$$

by virtue of (25) it easily follows that

$$
1+\left(U_{3}+W^{*}\right)^{2} \geqslant 1
$$

Choosing $\mu_{2}=\mu_{3}=1$, from (6) and (26), it follows that

$$
\begin{aligned}
\left\langle U_{1}, F_{1}^{*}\right\rangle \leqslant C_{1}\left\langle 1, U_{1}^{2}\right\rangle & +C_{2}\left\langle 1,\left|U_{1}\right|^{3}\right\rangle, \\
\left\langle\bar{A}_{1} U_{2}-\bar{A}_{3} U_{3}, F_{2}^{*}\right\rangle \leqslant & C_{3}\left\langle U_{1}^{2},\left|U_{2}\right|\right\rangle+C_{4}\left\langle\left|U_{2}\right|,\left|U_{3}\right|\right\rangle+C_{5}\left\langle\left|U_{1}\right|,\left|U_{2}\right|^{2}\right\rangle \\
& +C_{6}\left\langle U_{1}^{2},\left|U_{3}\right|\right\rangle+C_{7}\left\langle U_{3}^{2}, 1\right\rangle+C_{8}\left\langle U_{3}^{2},\left|U_{2}\right|\right\rangle, \\
\left\langle\bar{A}_{2} U_{3}-\bar{A}_{3} U_{2}, F_{3}^{*}\right\rangle \leqslant & C_{9}\left\langle\left|U_{1}\right|,\left|U_{3}\right|\right\rangle+C_{10}\left\langle\left|U_{1}\right|,\left|U_{2}\right|\right\rangle
\end{aligned}
$$

with

$$
\begin{aligned}
& C_{1}=\rho \delta, \quad C_{2}=\rho \mu_{1}, \quad C_{3}=\frac{\beta\left|\bar{A}_{1}\right| W^{*}}{\mu} \mu_{1}^{2}+\frac{\mu_{1}}{2}\left|\bar{A}_{3}\right|, \\
& C_{4}=\beta\left|\bar{A}_{1}\right|, \quad C_{5}=\mu_{1}\left|\bar{A}_{1}\right|, \quad C_{6}=\frac{\beta\left|\bar{A}_{3}\right| \mu_{1}^{2} W^{*}}{\mu}, \\
& C_{7}=\beta\left|\bar{A}_{3}\right|, \quad C_{8}=\frac{\mu}{2}\left|\bar{A}_{3}\right|, \quad C_{9}=k \mu_{1} \bar{A}_{2}, \quad C_{10}=k \mu_{1}\left|\bar{A}_{3}\right| .
\end{aligned}
$$

By virtue of (27)-(29) one obtains

$$
\begin{aligned}
\left|\bar{\Phi}_{3}\right| \leqslant & \left(C_{1}+\frac{C_{9}}{2}+\frac{C_{10}}{2}\right)\left\|U_{1}\right\|^{2}+\left(\frac{C_{4}}{2}+\frac{C_{10}}{2}\right)\left\|U_{2}\right\|^{2} \\
& +\left(\frac{C_{4}}{2}+C_{7}+\frac{C_{9}}{2}\right)\left\|U_{3}\right\|^{2}+C_{2}\left\langle 1,\left|U_{1}\right|^{3}\right\rangle+C_{3}\left\langle U_{1}^{2},\left|U_{2}\right|\right\rangle \\
& +C_{5}\left\langle\left|U_{1}\right|, U_{2}^{2}\right\rangle+C_{6}\left\langle U_{1}^{2},\left|U_{3}\right|\right\rangle+C_{8}\left\langle U_{3}^{2},\left|U_{2}\right|\right\rangle .
\end{aligned}
$$


The Hölder inequality implies

$$
\begin{aligned}
\left|\bar{\Phi}_{3}\right| \leqslant & \left(C_{1}+\frac{C_{9}}{2}+\frac{C_{10}}{2}\right)\left\|U_{1}\right\|^{2}+\left(\frac{C_{4}}{2}+\frac{C_{10}}{2}\right)\left\|U_{2}\right\|^{2} \\
& +\left(\frac{C_{4}}{2}+C_{7}+\frac{C_{9}}{2}\right)\left\|U_{3}\right\|^{2}+\left(\left\|U_{1}\right\|^{2}+\left\|U_{2}\right\|^{2}+\left\|U_{3}\right\|^{2}\right)^{1 / 2} \\
& \times\left[\left(C_{2}+C_{3}+C_{6}\right)\left\|U_{1}\right\|_{4}^{2}+\left(C_{5}+2\right)\left\|U_{2}\right\|_{4}^{2}+\left(C_{8}+2\right)\left\|U_{3}\right\|_{4}^{2}\right]
\end{aligned}
$$

and in view of the embedding inequality

$$
\|f\|_{4}^{2} \leqslant K\left(\|\nabla f\|^{2}+\|f\|^{2}\right), \quad K=K(\Omega)=\text { const }>0,
$$

it turns out that

$$
\begin{aligned}
\left|\bar{\Phi}_{3}\right| \leqslant & H_{1}\left\|U_{1}\right\|^{2}+H_{2}\left(\left\|U_{2}\right\|^{2}+\left\|U_{3}\right\|^{2}\right) \\
& +K M\left(\left\|\nabla U_{1}\right\|^{2}+\left\|\nabla U_{2}\right\|^{2}+\left\|\nabla U_{3}\right\|^{2}+\left\|U_{1}\right\|^{2}+\left\|U_{2}\right\|^{2}+\left\|U_{3}\right\|^{2}\right) \\
& \times\left(\left\|U_{1}\right\|^{2}+\left\|U_{2}\right\|^{2}+\left\|U_{3}\right\|^{2}\right)^{1 / 2}
\end{aligned}
$$

with

$$
\begin{aligned}
H_{1} & =C_{1}+\frac{C_{9}}{2}+\frac{C_{10}}{2}, \\
H_{2} & =\max \left\{\frac{C_{4}}{2}+\frac{C_{10}}{2}, \frac{C_{4}}{2}+C_{7}+\frac{C_{9}}{2}\right\}, \\
M & =\max \left\{C_{2}+C_{3}+C_{6}, C_{5}+2, C_{8}+2\right\} .
\end{aligned}
$$

Since $\bar{A}>0$, an inequality like (18) holds also for $\bar{W}$, with $K_{1}, K_{2}$ replaced by two positive constant $\bar{K}_{1}, \bar{K}_{2}$. Hence, on taking into account (30), (24) implies

$$
\begin{aligned}
\frac{\mathrm{d} \bar{W}}{\mathrm{~d} t} \leqslant & -\left[\left(\frac{1}{2}\left|\bar{b}_{11}\right|-H_{1}\right)\left\|U_{1}\right\|^{2}+\left(\frac{|\bar{I} \bar{A}|}{2}-H_{2}\right)\left(\left\|U_{2}\right\|^{2}+\left\|U_{3}\right\|^{2}\right)\right] \\
& -\left[\epsilon \bar{\delta}-K M\left(\left\|U_{1}\right\|^{2}+\frac{1}{\bar{K}_{2}} V\right)^{1 / 2}\right]\left(\left\|\nabla U_{1}\right\|^{2}+\left\|\nabla U_{2}\right\|^{2}+\left\|\nabla U_{3}\right\|^{2}\right) \\
& +K M\left(\left\|U_{1}\right\|^{2}+\frac{1}{\bar{K}_{2}} V\right)^{3 / 2},
\end{aligned}
$$

i.e.

$$
\frac{\mathrm{d} \bar{W}}{\mathrm{~d} t} \leqslant-\left(\delta_{1}-\delta_{2} \bar{W}^{1 / 2}\right) \bar{W}-\left(\delta_{3}-\delta_{4} \bar{W}^{1 / 2}\right)\left(\left\|\nabla U_{1}\right\|^{2}+\left\|\nabla U_{2}\right\|^{2}+\left\|\nabla U_{3}\right\|^{2}\right)
$$

with

$$
\begin{aligned}
& \delta_{1}=\inf \left\{\frac{1}{2}\left|\bar{b}_{11}\right|-H_{1}, \frac{1}{2 \bar{K}_{2}}\left(|\bar{I} \bar{A}|-H_{2}\right)\right\} \\
& \delta_{2}=K M\left(1+\frac{1}{\bar{K}_{2}}\right)^{3 / 2}, \quad \delta_{3}=\epsilon \bar{\delta}, \quad \delta_{4}=K M\left(1+\frac{1}{\bar{K}_{2}}\right)^{1 / 2} .
\end{aligned}
$$


By recursive argument

$$
\bar{W}_{0}<\inf \left[\left(\frac{\delta_{1}}{\delta_{2}}\right)^{2},\left(\frac{\delta_{3}}{\delta_{4}}\right)^{2}\right]
$$

implies

$$
\bar{W} \leqslant \bar{W}_{0} \mathrm{e}^{-\left(\delta_{1}-\delta_{2} \bar{W}_{0}^{1 / 2}\right) t} .
$$

We can summarize the results of this section in the following theorem.

Theorem 2. Let $\bar{b}_{11}<0$, (22) and

$$
\frac{1}{2}\left|\bar{b}_{11}\right|-H_{1}>0, \quad|\bar{I} \bar{A}|-H_{2}>0
$$

hold. Then the zero solution of (20) is asymptotically stable with respect to the $L^{2}(\Omega)$-norm.

\section{Absorbing set}

Let us consider the energy

$$
E=\frac{1}{2}\left(\|u\|^{2}+\|v\|^{2}+\|w\|^{2}\right)
$$

as a measure - in the phase space - of the distance from the origin and denote by $|\Omega|$ the measure of $\Omega$. Along the solutions of (2), belonging to $I_{2}$, one has

$$
\begin{aligned}
\frac{\mathrm{d} E}{\mathrm{~d} t}= & d_{1} \int_{\Omega} u \Delta u \mathrm{~d} \Omega+\int_{\Omega} v \Delta v \mathrm{~d} \Omega+d_{3} \int_{\Omega} w \Delta w \mathrm{~d} \Omega \\
& -\alpha \int_{\Omega} \nabla\left[\frac{u}{(1+v)^{2}} \nabla v\right] u \mathrm{~d} \Omega+\int_{\Omega} \rho u^{2}\left(\frac{\delta w^{2}}{1+w^{2}}-u\right) \mathrm{d} \Omega \\
& +\beta \int_{\Omega} w v \frac{u^{2}}{\mu+u^{2}} \mathrm{~d} \Omega-\int_{\Omega} u v^{2} \mathrm{~d} \Omega-k \int_{\Omega} u w \frac{w^{2}}{1+w^{2}} \mathrm{~d} \Omega .
\end{aligned}
$$

By virtue of the boundary conditions (3) and (4) it follows that

$$
\begin{aligned}
\frac{\mathrm{d} E}{\mathrm{~d} t} \leqslant & -d_{1} \bar{\alpha}\|u\|^{2}-\bar{\alpha}\|v\|^{2}-d_{3} \bar{\alpha}\|w\|^{2}-\alpha \int_{\Omega} \nabla\left[\frac{u}{(1+v)^{2}} \nabla v\right] u \mathrm{~d} \Omega \\
& +\rho \delta \int_{\Omega} u^{2}\left[1-\frac{1}{1+w^{2}}\right] \mathrm{d} \Omega-\rho \int_{\Omega} u^{3} \mathrm{~d} \Omega+\beta \int_{\Omega} w v \mathrm{~d} \Omega \\
& -\beta \mu \int_{\Omega} \frac{w v}{\mu+u^{2}} \mathrm{~d} \Omega-\int_{\Omega} u v^{2} \mathrm{~d} \Omega-k \int_{\Omega} u w \frac{w^{2}}{1+w^{2}} \mathrm{~d} \Omega .
\end{aligned}
$$


Moreover, in view of (3), one obtains

$$
\begin{aligned}
& -\alpha \int_{\Omega} \nabla\left[\frac{u}{(1+v)^{2}} \nabla v\right] u \mathrm{~d} \Omega \\
& =\alpha \int_{\Omega} \nabla\left[u \nabla(1+v)^{-1}\right] u \mathrm{~d} \Omega=-\alpha \int_{\Omega} u \nabla u \nabla(1+v)^{-1} \mathrm{~d} \Omega \\
& =\alpha \int_{\Omega} \nabla(u \nabla u)(1+v)^{-1} \mathrm{~d} \Omega \\
& =\alpha \int_{\Omega}(\nabla u)^{2}(1+v)^{-1} \mathrm{~d} \Omega+\alpha \int_{\Omega} u \Delta u(1+v)^{-1} \mathrm{~d} \Omega .
\end{aligned}
$$

Considering the biologically meaningful concentration $(u>0, v>0, w>0)$ one has that $1+v>1$ and in view of (31) one obtains

$$
-\alpha \int_{\Omega} \nabla\left[\frac{u}{(1+v)^{2}} \nabla v\right] u \mathrm{~d} \Omega \leqslant \alpha\|\nabla u\|^{2}+\alpha \int_{\Omega}|u \Delta u| \mathrm{d} \Omega \leqslant 0 .
$$

Since

$$
\rho \delta\|u\|^{2}-\rho\left\langle 1, u^{3}\right\rangle \leqslant \frac{\rho \delta}{2 \epsilon_{1}}\langle 1, u\rangle+\left(\frac{\rho \delta \epsilon_{1}}{2}-\rho\right)\left\langle 1, u^{3}\right\rangle
$$

with $\epsilon_{1}$ positive constant, choosing

$$
\epsilon_{1}=\frac{2}{\delta}
$$

it turns out that

$$
\frac{\mathrm{d} E}{\mathrm{~d} t} \leqslant-d_{1} \bar{\alpha}\|u\|^{2}-\bar{\alpha}\|v\|^{2}-d_{3} \bar{\alpha}\|w\|^{2}+\frac{\rho \delta^{2}}{4}\langle 1, u\rangle+\beta \int_{\Omega} v w \mathrm{~d} \Omega .
$$

On the other hand

$$
\beta\langle v, w\rangle \leqslant \frac{\beta}{2 \epsilon_{2}}\|v\|^{2}+\frac{\beta \epsilon_{2}}{2}\|w\|^{2}, \quad \frac{\rho \delta^{2}}{4}\langle 1, u\rangle \leqslant \frac{\rho^{2} \delta^{4}}{32 \epsilon_{3}}|\Omega|+\frac{\epsilon_{3}}{2}\|u\|^{2},
$$

therefore for

$$
\epsilon_{3}=d_{3} \bar{\alpha}
$$

one obtains

$$
\frac{\mathrm{d} E}{\mathrm{~d} t} \leqslant-\frac{d_{1} \bar{\alpha}}{2}\|u\|^{2}-\left(\bar{\alpha}-\frac{\beta}{2 \epsilon_{2}}\right)\|v\|^{2}-\left(d_{3} \bar{\alpha}-\frac{\beta \epsilon_{2}}{2}\right)\|w\|^{2}+\frac{\rho^{2} \delta^{4}}{32 d_{1} \bar{\alpha}}|\Omega|,
$$

i.e.

$$
\frac{\mathrm{d} E}{\mathrm{~d} t} \leqslant-C_{1}\left(\|u\|^{2}+\|v\|^{2}+\|w\|^{2}\right)+C_{2}
$$


with

$$
C_{1}=\inf \left\{\frac{d_{1} \bar{\alpha}}{2},\left(\bar{\alpha}-\frac{\beta}{2 \epsilon_{2}}\right),\left(d_{3} \bar{\alpha}-\frac{\beta \epsilon_{2}}{2}\right)\right\}, \quad C_{2}=\frac{\rho^{2} \delta^{4}}{32 d_{1} \bar{\alpha}}|\Omega| .
$$

Now, assuming

$$
\bar{\alpha}^{2} d_{3}>\frac{\beta^{2}}{4}
$$

and choosing $\epsilon_{2}$ in such a way that

$$
\frac{\beta}{2 \bar{\alpha}}<\epsilon_{2}<\frac{2 d_{3} \bar{\alpha}}{\beta}
$$

it follows that

$$
\frac{\mathrm{d}}{\mathrm{d} t} E(t)+2 C_{1} E(t) \leqslant C_{2}
$$

with $C_{1}, C_{2}$ positive constants independent of the initial data $\left(u_{0}(x), v_{0}(x), w_{0}(x)\right)$.

Hence the following theorem holds

Theorem 3. Let (32) holds. Then it turns out that for any $\bar{\delta}>0$, the ball $S(\bar{\delta})$ centered at the origin $(0,0)$ defined by

$$
S(\bar{\delta})=\left\{(u, v, w): E \leqslant(1+\bar{\delta}) \frac{C_{2}}{2 C_{1}}\right\}
$$

is an absorbing set.

Proof. First of all we observe that $S(\bar{\delta})$ is invariant, in fact from

$$
E(\bar{t})=(1+\bar{\delta}) \frac{C_{2}}{2 C_{1}},
$$

by virtue of (33) one obtains

$$
\left(\frac{\mathrm{d} E}{\mathrm{~d} t}\right)_{t=\bar{t}}<C_{2}-2 C_{1}(1+\bar{\delta}) \frac{C_{2}}{2 C_{1}}<-\bar{\delta} C_{2}<0 .
$$

Moreover from (33) it turns out that

$$
E(t) \leqslant E(0) \mathrm{e}^{-2 C_{1} t}+\frac{C_{2}}{2 C_{1}} .
$$

Thus, denoting by $\Gamma$ a bounded set of the phase-space, there exists a positive constant $M$ such that

$$
\sup _{\Gamma} E(t) \leqslant M
$$

From

$$
M \mathrm{e}^{-2 C_{1} t}+\frac{C_{2}}{2 C_{1}}=(1+\bar{\delta}) \frac{C_{2}}{2 C_{1}}
$$

we obtain that, for any $t>t^{*}$ with

$$
t^{*}=\frac{1}{2 C_{1}} \log \frac{2 M C_{1}}{\bar{\delta} C_{2}},
$$

any trajectory starting initially in $\Gamma$, belongs to $S(\bar{\delta})$. 


\section{Global nonlinear asymptotic stability}

If one is able to "control" the functional $\Phi_{3}$ in (12), then conditions for the global stability may be obtained. This is the main goal of this section.

By virtue of the ultimately boundedness $L^{2}$-norm of the solutions of (2), it follows that we can assume that exists a positive constant $H$ such that

$$
\left|U_{1}\right|,\left|U_{2}\right|,\left|U_{3}\right|<H \quad \text { a.e. in } \Omega \times R^{+} .
$$

In this way, by virtue of (27)-(29), we get the estimate

$$
\left|\Phi_{3}\right| \leqslant M_{1}\left\|U_{1}\right\|^{2}+M_{2}\left(\left\|U_{2}\right\|^{2}+\left\|U_{3}\right\|^{2}\right)
$$

with

$$
\begin{aligned}
& M_{1}=\tilde{C}_{1}+\left(\tilde{C}_{2}+\tilde{C}_{3}+\tilde{C}_{6}\right) H+\frac{\tilde{C}_{9}}{2}+\frac{\tilde{C}_{10}}{2} \\
& M_{2}=\max \left\{\frac{\tilde{C}_{4}}{2}+\tilde{C}_{5} H+\frac{\tilde{C}_{10}}{2}, \frac{\tilde{C}_{4}}{2}+\tilde{C}_{7}+\tilde{C}_{8} H+\frac{\tilde{C}_{9}}{2}\right\}
\end{aligned}
$$

and

$$
\begin{aligned}
& \tilde{C}_{1}=\rho \delta, \quad \tilde{C}_{2}=\rho \mu_{1}, \quad \tilde{C}_{3}=\frac{\beta\left|A_{1}\right| W^{*}}{\mu} \mu_{1}^{2}+\frac{\mu_{1}}{2}\left|A_{3}\right|, \\
& \tilde{C}_{4}=\beta\left|A_{1}\right|, \quad \tilde{C}_{5}=\mu_{1}\left|A_{1}\right|, \quad \tilde{C}_{6}=\frac{\beta\left|A_{3}\right| \mu_{1}^{2} W^{*}}{\mu}, \\
& \tilde{C}_{7}=\beta\left|A_{3}\right|, \quad \tilde{C}_{8}=\frac{\mu}{2}\left|A_{3}\right|, \quad \tilde{C}_{9}=k \mu_{1} A_{2}, \quad \tilde{C}_{10}=k \mu_{1}\left|A_{3}\right| .
\end{aligned}
$$

Hence the following theorem holds

Theorem 4. Let (13), (15), (32) and

$$
\frac{1}{2}\left|b_{11}\right|-M_{1}>0, \quad|I A|-M_{2}>0
$$

hold. Then the zero solution of (11) is globally asymptotically stable with respect to the $L^{2}$-norm.

Proof. On taking into account (14), (16) and (18), (12) implies

$$
\begin{aligned}
\dot{W} \leqslant & -\frac{1}{2}\left|b_{11}\right|\left\|U_{1}\right\|^{2}-\frac{1}{2}|I A|\left(\left\|U_{2}\right\|^{2}+\left\|U_{3}\right\|^{2}\right) \\
& +M_{1}\left\|U_{1}\right\|^{2}+M_{2}\left(\left\|U_{2}\right\|^{2}+\left\|U_{3}\right\|^{2}\right),
\end{aligned}
$$

i.e.

$$
\dot{W} \leqslant-\chi_{1} W
$$

with

and hence

$$
\chi_{1}=\inf \left\{\frac{1}{2}\left|b_{11}\right|-M_{1}, \frac{1}{2 K_{2}}\left(|I A|-2 M_{2}\right)\right\}
$$

$$
W(t) \leqslant W(0) \mathrm{e}^{-\chi_{1} t}
$$




\section{Appendix. Proof of Lemma 1}

Since the invariants $I_{1}, I_{2}, I_{3}$ of

$$
\left(\begin{array}{ccc}
\gamma_{11} & 0 & 0 \\
\gamma_{21} & \gamma_{22} & \gamma_{23} \\
\gamma_{31} & \gamma_{32} & \gamma_{33}
\end{array}\right)
$$

and $I_{1} I_{2}-I_{3}$ are given by

$$
\begin{aligned}
& I_{1}=\gamma_{11}+I, \quad I_{2}=\gamma_{11} I+A, \quad I_{3}=\gamma_{11} A, \\
& I_{1} I_{2}-I_{3}=\gamma_{11} I\left(I+\frac{\gamma_{11}^{2}+A}{\gamma_{11}}\right),
\end{aligned}
$$

it immediately follows that (10) imply the Routh-Hurwitz stability condition for (35)

$$
I_{1}<0, \quad I_{3}<0, \quad I_{1} I_{2}-I_{3}<0 .
$$

Vice versa, let (37) hold. Since one easily verifies that $\gamma_{11}$ is a real root of (35), by virtue of (37), it follows that $\gamma_{11}<0$.

Then (36) $)_{3}$ and (37) 2 imply $A>0$. It remains to obtain $I<0$. But (37) implies

$$
\left(-\gamma_{11}\right) I\left(I+\frac{\gamma_{11}^{2}+A}{\gamma_{11}}\right)=\left(-\gamma_{11}\right) I^{2}-\left(\gamma_{11}^{2}+A\right) I>0,
$$

and the roots of

$$
\left(-\gamma_{11}\right) I^{2}-\left(\gamma_{11}^{2}+A\right) I=0
$$

are 0 and $\left(\gamma_{11}^{2}+A\right) /-\gamma_{11}>0$, hence $I \notin\left[0,-\left(\gamma_{11}^{2}+A\right) / \gamma_{11}\right]$.

Since $-\left(\gamma_{11}^{2}+A\right) / \gamma_{11}>-\gamma_{11},(37)_{1}$ does not allow, in view of (36) $)_{1}, I>-\gamma_{11}$, hence $I<0$.

\section{References}

1. R. Tyson, S.R. Lubkin, J.D. Murray, A minimal mechanism for bacterial pattern formation, Proc. R. Soc. Lond., Ser. B, 266(1416):299-304, 1999.

2. E.O. Budrene, H.C. Berg, Complex patterns formed by motile cells of Escherichia coli, Nature, 349:630-633, 1991.

3. E.O. Budrene, H.C. Berg, Dynamics of formation of symmetrical patterns by chemotactic bacteria, Nature, 376:49-53, 1995.

4. K. Kawasaki, A. Mochizuki, M. Matsushita, T. Umeda, N. Shigesada, Modeling spatiotemporal patterns generated by Bacillus Subtilis, J. Theor. Biol., 188(2):177-185, 1997.

5. E.F. Keller, L.A. Segel, Model for chemotaxis, J. Theor. Biol., 30(2):225-234, 1971. 
6. J.D. Murray, Mathematical Biology, 2nd edition, Biomath., Berl., Vol. 19, Springer-Verlag, Berlin, 1993.

7. T. Hillen, K.J. Painter, A user's guide to PDE models for chemotaxis, J. Math. Biol., 58(12):183-217, 2009.

8. J. Smoller, Shock Waves and Reaction-Diffusion Equations, A Series of Comprehensive Studies in Mathematics, Vol. 258, Springer-Verlag, New York, 1983.

9. J.N. Flavin, S. Rionero, Qualitative Estimates for Partial Differential Equation: An Introduction, CRC Press, Boca Raton, FL, 1995.

10. R. Baronas, R. Šimkus, Modeling the bacterial self-organization in a circular container along the contact line as detected by bioluminescence imaging, Nonlinear Anal. Model Control, 16(3):270-282, 2011.

11. R. Čiegis, A. Bugajev, Numerical approximation of one model of bacterial self-organization, Nonlinear Anal. Model Control, 17(3):253-270, 2012.

12. R. Tyson, S.R. Lubkin, J.D. Murray, Model and analysis of chemotactic bacterial patterns in a liquid medium, J. Math. Biol., 38(4):359-375, 1999.

13. S. Rionero, Stability of ternary reaction diffusion dynamical systems, Atti Accad. Naz. Lincei, Cl. Sci. Fis. Mat. Nat., IX. Ser., Rend. Lincei, Mat. Appl., 22(3):245-268, 2011.

14. S. Rionero, A rigorous reduction of the $L^{2}$-stability of the solutions to a nonlinear binary reaction-diffusion system of PDEs to the stability of the solutions to a linear binary system of ODEs, J. Math. Anal. Appl., 319(2):377-397, 2006.

15. J.N. Flavin, S. Rionero, Cross-diffusion influence on the nonlinear $L^{2}$-stability analysis for the Lotka-Volterra reaction-diffusion system of PDEs, IMA J. Appl. Math., 72(5):540-555, 2007.

16. S. Rionero, Long time behaviour of three competing species and mututalistic communities, in T. Ruggeri, M. Sammartino (Eds.) Asymptotic Methods in Nonlinear Wave Phenomena: In Honor of the 65th Birthday of Antonio Greco, Palermo, Italy, 5-7 June, 2006, World Scientific, Singapore, 2007, pp. 171-185.

17. S. Rionero, On the reducibility of the $L^{2}$-stability of ternary reaction diffusion systems of PDEs, in Proceedings "WASCOM 2009" 15th Conference on Waves and Stability in Continuous Media, World Scientific, Singapore, 2010, pp. 321-331.

18. S. Rionero, Long-time behaviour of multi-component fluid mixture in porous media, Int. $J$. Eng. Sci., 48(11):1519-1533, 2010 